Pacific Journal of Mathematic 


\title{
A THIRD ORDER IRREGULAR BOUNDARY VALUE PROBLEM AND THE ASSOCIATED SERIES
}

\author{
GEORGE SEIFERT
}

1. Introduction. Certain problems in aeroelastic wing theory [1] give rise to a third order irregular boundary value problem of the form given in equation (1) below. Questions have been raised [1] as to conditions under which functions have an expansion in terms of the associated characteristic functions. It is shown in this paper that the general approach by L. E. Ward [2] in dealing with a somewhat more specialized problem can be suitably modified to provide an answer to these questions.

We are concerned with the differential boundary value problem

$$
\begin{aligned}
& L(u(x), \lambda)=u^{\prime \prime \prime}(x)+p(x) u^{\prime}(x)+((x)+\lambda) u(x)=0, \\
& u(0)=u^{\prime}(0)=u^{\prime \prime}(1)=0,
\end{aligned}
$$

where $p(x)=x \psi_{1}\left(x^{3}\right), q(x)=\psi_{2}\left(x^{3}\right)$, and $\psi_{1}(z)$ and $\psi_{2}(z)$ are real for real $z$ and analytic on $|z| \leq 1$. We seek conditions on $f(x)$ such that it be expansible in terms of the characteristic functions of (1) and its adjoint.

We shall first need a number of definitions and lemmas. Define:

$$
\begin{aligned}
& \delta_{3}(t) \equiv e^{\omega_{1} t}-\omega_{2} e^{\omega_{2} t}-\omega_{3} e^{\omega_{3} t}, \\
& \delta_{2}(t) \equiv-\delta_{3}^{\prime}(t), \\
& \delta_{1}(t) \equiv-\delta_{2}^{\prime}(t),
\end{aligned}
$$

where $\omega_{1}=-1, \omega_{2}=e^{\pi i / 3}, \omega_{3}=e^{-\pi i / 3}$;

$$
\Delta(x, t, \rho) \equiv \rho^{-1} \delta_{3}[\rho(x-t)] r(t)-\delta_{2}[\rho(x-t)] p(t)
$$

where $r(t)=q(t)-p^{\prime}(t)$, and the complex number $\rho$ satisfies

$$
\rho^{3}=\lambda,|\arg \rho| \leq \pi / 3
$$

Received October 8, 1951. The author wishes to thank Professor L. K. Jackson for helpful suggestions towards clarification of the notation used.

Pacific J. Math . 2 (1952), 395-406 
iii) the regions $S_{1}$ and $S_{2}$ of the $\rho$-plane by $0 \leq \arg \rho \leq \pi / 3$ and $-\pi / 3 \leq$ arg $\rho \leq 0$, respectively.

We shall be concerned with the integral equation

$$
u(x, \xi, \rho)=\delta_{3}[\rho(x-\xi)]-\frac{1}{3 \rho} \int_{\xi}^{x} \Delta(x, t, \rho) u(t, \xi, \rho) d t .
$$

2. Lemmas. We shall use the following results.

LEMMA 1. Equation (2) has for fixed $\rho$ a unique solution analytic in $x$ and in $\xi$ on $|x| \leq 1$ and $|\xi| \leq 1$, respectively, where $x$ and $\xi$ are complex variables. ${ }^{1}$

Proof. For fixed $\rho$, define

$$
\begin{aligned}
& f_{1}(x, \xi) \equiv \delta_{3}[\rho(x-\xi)], \\
& f_{j}(x, \xi) \equiv-\frac{1}{3 \rho} \int_{\xi}^{x} \Delta(x, t, \rho) f_{j-1}(t, \xi) d t .
\end{aligned}
$$

Then

$\left|f_{1}(x, \xi)\right| \leq M$

$\left|f_{2}(x, \xi)\right|=\left|-\frac{1}{3 \rho} \int_{\xi}^{x} \Delta(x, t, \rho) f_{1}(t, \xi) d t\right|<M N \int_{\xi}^{x}|d t|=M N|x-\xi|$.

Hence, by induction,

$$
\left|f_{j}(x, \xi)\right|<\frac{M N^{j-1}|x-\xi|^{j-1}}{(j-1) !} \quad(j=2,3,4, \cdots)
$$

consequently,

$$
\sum_{j=1}^{\infty} f_{j}(x, \xi)=w(x, \xi)
$$

where $w(x, \xi)$ is analytic in $x$ and in $\xi$ in $|x| \leq 1$ and $|\xi| \leq 1$, respectively. By direct substitution into (2), we see that $w(x, \xi)$ is a solution.

To show uniqueness, consider

1 The variables $x$ and $\xi$ will always be considered real, unless otherwise indicated, as here; in this case, as in subsequent cases, integration between complex limits, as in equation (2), may be taken along a straight line in the complex plane. 


$$
z(x, \xi)=u_{1}(x, \xi)-u_{2}(x, \xi)
$$

where $u_{1}(x, \xi)$ and $u_{2}(x, \xi)$ are solutions of (2). Clearly $z(x, \xi)$ must satisfy the equation

$$
z(x, \xi)=-\frac{1}{3 \rho} \int_{\xi}^{x} \Delta(x, t, \xi) z(t, \xi) d t
$$

and for real $x$ and $\xi, z(x, \xi)$ is easily seen to satisfy the system ${ }^{2}$

$$
L(z(x, \xi), \lambda)=0, \quad z(\xi, \xi)=z^{\prime}(\xi, \xi)=z^{\prime \prime}(\xi, \xi)=0
$$

Hence $z(x, \xi)=0$ identically in $x$ for any fixed $\xi$, for real $x$ and $\xi$; this implies $z(x, \xi)=0$ identically for complex $x$ and $\xi$ and completes the proof.

LEMMA 2. For real $x$ and $\xi$, (2) is equivalent to the system

$$
L(u(x, \xi), \lambda)=0, u(\xi, \xi)=u^{\prime}(\xi, \xi)=0, u^{\prime \prime}(\xi, \xi)=3 \rho^{2}
$$

Proof. Substitution in $(2 \mathrm{a})$ of $u(x, \xi, \rho)$ as given by (2) shows that the unique solution of (2) is a solution of (2a). However, for fixed $\xi$ and $\rho$, (2a) also has a unique solution. Clearly, these unique solutions must coincide, and our proof is complete.

LemMA 3. Let $u(x, \xi, \rho)$ be a solution of (2). Then ${ }^{3}$

a)

$$
u(x, \xi, \rho)=e^{\omega_{3} \rho(x-\xi)} E(x, \xi, \rho)
$$

provided $|\rho|$ is large enough $\rho \in S_{1}, x \geq \xi$;

b)

$$
\begin{aligned}
& u\left(-\omega_{2} x,-\omega_{2} \xi, \rho\right)=-\omega_{3} u(x, \xi, \rho) ; \\
& u^{\prime \prime}(1,0, \rho)=\rho^{2} e^{\omega_{3} \rho} M(\rho),
\end{aligned}
$$

c)

where $|M(\rho)| \geq m>0$, provided

$$
\rho=\frac{2 n+2}{\sqrt{3}} \pi e^{i \theta}
$$

2 Unless otherwise indicated, the prime will always denote differentiation with respect to the first indicated variable.

3 Functions of $\rho$ and other variables which are bounded for $|\rho|$ sufficiently large will be denoted by $E()$. 
for sufficiently large $n$.

Proof of a). As in Lemma 2 of [3], p. 211, it follows that for $\rho \in S_{1}$, we have

$$
u(x, \xi, \rho)=e^{\omega_{3} \rho(x-\xi)}\left[-\omega_{3}-\omega_{2} e^{\left(\omega_{2}-\omega_{3}\right) \rho(x-\xi)}+z(x, \xi, \rho)\right],
$$

where $|z(x, \xi, \rho)|<M$ for $|\rho|$ sufficiently large and $x \geq \xi$. Hence

$$
u(x, \xi, \rho)=e^{\omega_{3} \rho(x-\xi)} E(x, \xi, \rho) .
$$

Proof of b ). Using (2), we have

$$
\begin{aligned}
u\left(-\omega_{2} x,-\omega_{2} \xi, \rho\right) & =\delta_{3}\left[-\omega_{2} \rho(x-\xi)\right] \\
& -\frac{1}{3 \rho} \int_{-\omega_{2} \xi}^{-\omega_{2} x} \Delta\left(-\omega_{2} x, s, \rho\right) u\left(s,-\omega_{2} \xi, \rho\right) d s \\
=-\omega_{3} \delta_{3}[\rho(x-\xi)] & \\
& +\frac{\omega_{2}}{3 \rho} \int_{\xi}^{x} \Delta\left(-\omega_{2} x,-\omega_{2} t, \rho\right) u\left(-\omega_{2} t,-\omega_{2} \xi, \rho\right) d t .
\end{aligned}
$$

But

$$
\begin{aligned}
\Delta\left(-\omega_{2} x,-\omega_{2} t, \rho\right) & =-\frac{\omega_{3}}{\rho} \delta_{3}[\rho(x-t)] r(t) \\
& +\omega_{2} \delta_{2}[\rho(x-t)]\left(-\omega_{2} p(t)\right)=-\omega_{3} \Delta(x, t, \rho) .
\end{aligned}
$$

Hence

$$
\begin{aligned}
u\left(-\omega_{2} x,-\omega_{2} \xi, \rho\right) & =-\omega_{3} \delta_{3}[\rho(x-\xi)] \\
& -\frac{1}{3 \rho} \int_{\xi}^{x} \Delta(x, t, \rho) u\left(-\omega_{2} t,-\omega_{2} \xi, \rho\right) d t
\end{aligned}
$$

Multiplying this last equation by $-\omega_{2}$, we have

$$
z(x, \xi, \rho)=\delta_{3}[\rho(x-\xi)]-\frac{1}{3 \rho} \int_{\xi}^{x} \Delta(x, t, \rho) z(t, \xi, \rho) d t,
$$


where

$$
z(x, \xi, \rho)=-\omega_{2} u\left(-\omega_{2} x,-\omega_{2} \xi, \rho\right)
$$

But by the uniqueness of the solutions of (2), we have

$$
-\omega_{2} u\left(-\omega_{2} x,-\omega_{2} \xi, \rho\right)=u(x, \xi, \rho)
$$

upon multiplication by $-\omega_{3}$, this gives $\left.b\right)$.

Proof of c ). We have, from (2),

$$
\begin{aligned}
u^{\prime \prime}(1,0, \rho) & =\rho^{2}\left[\delta_{1}(\rho)+\frac{e^{\omega_{3} \rho}}{\rho} E_{1}(\rho)\right] \\
& =\rho^{2} e^{\omega_{3} \rho}\left[1+e^{\left(\omega_{2}-\omega_{3}\right) \rho}+\frac{E_{2}(\rho)}{\rho}\right]
\end{aligned}
$$

for $\rho \in S_{1}$. Let $\rho=x+i y$, and define $\Phi(\rho)$ and $r_{n}$ by

$$
\Phi(\rho)=1+e^{\left(\omega_{2}-\omega_{3}\right) \rho} \text { and } r_{n}=\frac{2(n+1)}{\sqrt{3}} \pi,
$$

respectively. With $p=\left[3\left(r_{n}^{2}-x^{2}\right)\right]^{1 / 2}$, we have

$$
|\Phi(\rho)| \geq\left|1+e^{-p} \cos (\sqrt{3} x)\right|
$$

provided $\rho=r_{n} e^{i \theta}$ where $0 \leq 0 \leq \pi / 3$, and will show that

$$
e^{-p} \cos (\sqrt{3} x)>-\frac{1}{2} \text { for } \frac{r_{n}}{2} \leq x \leq r_{n}
$$

Since

$$
\cos (\sqrt{3} x) \geq 0 \quad \text { for } \quad r_{n}-\frac{\pi}{2 \sqrt{3}} \leq x \leq r_{n},
$$

it is clearly sufficient to show that

$$
e^{-p} \cos (\sqrt{3} x)>-\frac{1}{2} \text { for } \frac{r_{n}}{2} \leq x \leq r_{n}-\frac{\pi}{2 \sqrt{3}}
$$

Accordingly, we note that for $x$ in this interval, we have 


$$
e^{-p}|\cos (\sqrt{3} x)|<\frac{1}{p} \leq \frac{1}{\sqrt{3}\left[r_{n}^{2}-\left(r_{n}-\frac{\pi}{2 \sqrt{3}}\right)^{2}\right]^{1 / 2}}<\frac{1}{2}
$$

for all $n>N$, provided $N$ is sufficiently large. Taking $N$ large enough we also have

$$
\left|\frac{E_{2}(\rho)}{\rho}\right|<\frac{1}{4} \text { for } \rho=r_{n} e^{i \theta} \quad(0 \leq \theta \leq \pi / 3) .
$$

Hence

$$
\left|\Phi(\rho)+\frac{E_{2}(\rho)}{\rho}\right| \geq|\Phi(\rho)|-\left|\frac{E_{2}(\rho)}{\rho}\right|>\frac{1}{2}-\frac{1}{4}=\frac{1}{4} .
$$

This completes the proof of the lemma.

By the formal series for $f(x)$, we shall mean the series

$$
\sum_{k=1}^{\infty} a_{k} u_{k}(x) \text { where } a_{k}=\int_{0}^{1} f(x) v_{k}(x) d x / \int_{0}^{1} u_{k}(x) v_{k}(x) d x
$$

in which $u_{k}(x)$ and $v_{k}(x)$ are respectively the characteristic functions of the system (1) and its adjoint corresponding to the characteristic value $\lambda_{k}$.

Lemma 4. The sum of the first $n$ terms of the formal series for $f(x)$ is given by

$$
\begin{aligned}
I_{n}(x)=\frac{1}{2 \pi i} \int_{\gamma_{n}}\left[\int_{0}^{x} f(\xi) u(x, \xi, \rho) d \xi\right. \\
\left.\quad-\frac{u(x, 0, \rho)}{u^{\prime \prime}(1,0, \rho)} \int_{0}^{1} f\{\xi) u^{\prime \prime}(1, \xi, \rho) d \xi\right] d \rho \\
=\frac{1}{2 \pi i} \int_{\gamma_{n}}\left[\sigma(x)-\frac{u(x, 0, \rho)}{u^{\prime \prime}(1,0, \rho)} \sigma^{\prime \prime}(1)\right] d \rho,
\end{aligned}
$$

where $\sigma(x)=\int_{0}^{x} f(\xi) u(x, \xi, \rho) d \xi$, and $\gamma_{n}$ is the arc of the $\rho$-plane given by

$$
\rho=\frac{2 n+2}{\sqrt{3}} \pi e^{i \theta},-\pi / 3 \leq \theta \leq \pi / 3,
$$


the $\rho$ integration proceeding in a counter-clockwise direction.

We omit the proof of this lemma, as its details almost duplicate the discussion in [2], pp.424-426. We point out, however, that Lemma 2 is required in this proof.

Lemma 5. The function $\sigma(x)$ defined in the previous lemma satisfies the equation

$$
\sigma(x)=\int_{0}^{x} f(\xi) \delta_{3}[\rho(x-\xi)] d \xi-\frac{1}{3 \rho} \int_{0}^{x} \Delta(x, t, \rho) \sigma(t) d t
$$

furthermore, $\sigma(x)$ is its unique solution, is analytic on $0 \leq x \leq 1$, and can be put into the form

$$
\sigma(x)=u(x, 0, \rho) \Psi_{1}(\rho)+\Psi_{2}(x, \rho),
$$

where

$$
\Psi_{2}(x, \rho)=\frac{3 f(x)}{\rho}+\frac{E_{1}(x, \rho)}{\rho^{2}}, E_{1}^{\prime \prime}(x, \rho)=\rho^{2} E_{2}(x, \rho),
$$

provided $f(x)=x^{2} \phi\left(x^{3}\right)$, where $\phi(z)$ is analytic on $|z| \leq 1$.

Proof. Using (2) in the expression for $\sigma(x)$, we obtain

$$
\begin{aligned}
\sigma(x)=\int_{0}^{x} f(\xi) \delta_{3}[\rho(x-\xi)] d \xi \\
-\frac{1}{3 \rho} \int_{0}^{x} f(\xi) \int_{\xi}^{x} \Delta(x, t, \rho) u(t, \xi, \rho) d t d \xi \\
=\int_{0}^{x} f(\xi) \delta_{3}[\rho(x-\xi)] d \xi-\frac{1}{3 \rho} \int_{0}^{x} \Delta(x, t, \rho) \sigma(t) d t
\end{aligned}
$$

on changing the order of integration in the second integral. Uniqueness of the solution $\sigma(x)$ can be shown in the usual manner. (See the proof of Lemma 1.) We next substitute $u(x, 0, \rho) \Psi_{1}(\rho)+\Psi_{2}(x, \rho)$ into (3) for $\sigma(x)$, and obtain

$$
\begin{aligned}
& u(x, 0, \rho) \Psi_{1}(\rho)+\Psi_{2}(x, \rho)=\int_{0}^{x} f(\xi) \delta_{3}[\rho(x-\xi)] d \xi \\
& -\frac{\Psi_{1}(\rho)}{3 \rho} \int_{0}^{x} \Delta(x, t, \rho) u(t, 0, \rho) d t-\frac{1}{3 \rho} \int_{0}^{x} \Delta(x, t, \rho) \Psi_{2}(t, \rho) d t,
\end{aligned}
$$


Using (2) with $\xi=0$, and subtracting the term $u(x, 0, \rho) \Psi_{1}(\rho)$ from both sides, we obtain

$$
\begin{array}{r}
\Psi_{2}(x, \rho)=\int_{0}^{x} f(\xi) \delta_{3}[\rho(x-\xi)] d \xi-\Psi_{1}(\rho) \delta_{3}(\rho x) \\
-\frac{1}{3 \rho} \int_{0}^{x} \Delta(x, t, \rho) \Psi_{2}(t, \rho) d t
\end{array}
$$

On integrating by parts twice, we obtain

$$
\begin{gathered}
\int_{0}^{x} f(\xi) \delta_{3}[\rho(x-\xi)] d \xi=\frac{3 f(x)}{\rho}+\rho^{-2} \int_{0}^{x} f^{\prime \prime}(\xi) \delta_{2}[\rho(x-\xi)] d \xi \\
=\frac{3 f(x)}{\rho}+\rho^{-2} \delta_{3}(\rho x) \int_{0}^{y} f^{\prime \prime}(\xi) e^{\rho \xi} d \xi+\mathcal{L}_{3} f^{\prime \prime}(\xi) e^{\rho \xi} d \xi
\end{gathered}
$$

where $y$ is a complex number to be determined later, and

$$
\begin{array}{r}
\mathcal{L}_{3} F(t) d t=e^{\omega_{1} \rho x} \int_{y}^{x} F(t) d t \quad-\omega_{2} e^{\omega_{2} \rho x} \int_{y}^{-\omega_{2} x} F(t) d t \\
-\omega_{3} e^{\omega_{3} \rho x} \int_{y}^{-\omega_{3} x} F(t) d t .
\end{array}
$$

It is in this step that we use the form of $f(x)$ as stated in the hypothesis of this lemma; for the details, see [2], pp.428-429.

We also have

$$
\begin{aligned}
& \int_{0}^{x} \Delta(x, t, \rho) \Psi_{2}(t, \rho) d t=\frac{1}{\rho} \int_{0}^{x} \delta_{3}[\rho(x-t)] r(t) \Psi_{2}(t, \rho) d t \\
&+\int_{0}^{x} \delta_{2}[\rho(x-t)] p(t) \Psi_{2}(t, \rho) d t \\
&=\frac{\delta_{3}(\rho x)}{\rho} \int_{0}^{y} r(t) e^{\rho t} \Psi_{2}(t, \rho) d t+\mathcal{L}_{3} r(t) e^{\rho t} \Psi_{2}(t, \rho) d t \\
&+\delta_{3}(\rho x) \int_{0}^{x} p(t) e^{\rho t} \Psi_{2}(t, \rho) d t+\mathcal{L}_{3} p(t) e^{\rho t} \Psi_{2}(t, \rho) d t
\end{aligned}
$$




$$
=\delta_{3}(\rho x) \int_{0}^{y} R(t) e^{\rho t} \Psi_{2}(t, \rho) d t+\mathcal{L}_{3} R(t) e^{\rho t} \Psi_{2}(t, \rho) d t,
$$

where $R(t)=r(t) / \rho+p(t)$, and where we have made use of the properties of $p(t)$ and $r(t)$, and the fact that, from the form of $\Psi_{2}(t, \rho)$ in terms of $u(x$, $0, \rho)$ and Lemma 3, part b, we have

$$
\Psi_{2}\left(-\omega_{2} t, \rho\right)=-\omega_{3} \Psi_{2}(t, \rho) .
$$

Putting these results into equation (4), we obtain

$$
\begin{aligned}
& \Psi_{2}(x, \rho)=\frac{3 f(x)}{\rho}+ \delta_{3}(\rho x)\left[\Psi_{1}(\rho)-\right. \\
&-\frac{1}{\rho^{2}} \int_{0}^{y} f^{\prime \prime}(\xi) e^{\rho \xi} d \xi \\
&\left.+\frac{1}{3 \rho} \int_{0}^{y} R(t) e^{\rho t} \Psi_{2}(t, \rho) d t\right] \\
&+\frac{1}{\rho^{2}} \mathcal{L}_{3} f^{\prime \prime}(t) e^{\rho t} d t-\frac{1}{3 \rho} \mathcal{L}_{3} R(t) e^{\rho t} \Psi_{2}(t, \rho) d t .
\end{aligned}
$$

This equation will certainly be satisfied if

$$
\Psi_{2}(x, \rho)=\frac{3 f(x)}{\rho}+\frac{1}{\rho^{2}} \mathcal{L}_{3} f^{\prime \prime}(t) e^{\rho t} d t+\frac{1}{3 \rho} \mathcal{L}_{3} R(t) e^{\rho t} \Psi_{2}(t, \rho) d t
$$

and

$$
\Psi_{1}(\rho)=\frac{1}{\rho^{2}} \int_{0}^{y} f^{\prime \prime}(\xi) e^{\rho \xi} d \xi-\frac{1}{3 \rho} \int_{0}^{y} R(t) e^{\rho t} \Psi_{2}(t, \rho) d t .
$$

The proof of the existence of a unique solution $\Psi_{2}(x, \rho)$ of (5) will follow along the lines of the corresponding proof in [2], provided we can show that an expression of the form $\left|\mathcal{L}_{3} F(t) e^{\rho t} d t\right|$ is bounded for complex $\rho$ and $0 \leq$ $x \leq 1$ whenever $|F(z)|$ is on $|z| \leq 1$ and we take $y=-e^{-i \arg \rho}$. For we have

$$
\begin{aligned}
& \left|\mathcal{L}_{3} F(t) e^{\rho t} d t\right| \leq\left|e^{\omega_{1} \rho x}\right| \int_{y}^{x}|F(t)|\left|e^{\rho t}\right||d t| \\
& \quad+\left|e^{\omega_{2} \rho x}\right| \int_{y}^{-\omega_{2} x}|F(t)|\left|e^{\rho t}\right||d t|+\left|e^{\omega_{3} \rho x}\right| \int_{y}^{-\omega_{3} x}|F(t)|\left|e^{\rho t}\right||d t|
\end{aligned}
$$




$$
\begin{aligned}
& \leq \mu\left[\left|e^{\omega_{1} \rho x} \int_{y}^{x}\right| e^{\rho t}|| d t \mid\right. \\
& \left.\quad+\left|e^{\omega_{2} \rho x}\right| \int_{y}^{-\omega_{2} x}\left|e^{\rho t}\right||d t|+\left|e^{\omega_{3} \rho x}\right| \int_{y}^{-\omega_{3} x}\left|e^{\rho t}\right||d t|\right],
\end{aligned}
$$

where $|F(z)| \leq \mu$ on $|z| \leq 1$; and since each integrand in this last expression assumes its maximum at its upper limit, we have

$$
\left|\mathcal{L}_{3} F(t) e^{\rho t} d t\right| \leq 6 \mu
$$

We omit the rest of this existence proof. (See [2], pp.429-430.)

For the asymptotic form of $\Psi_{2}(x, \rho)$, we substitute

$$
\Psi_{2}(x, \rho)=\frac{3 f(x)}{\rho}+v(x, \rho)
$$

into (5). We obtain

$$
\begin{aligned}
v(x, \rho)= & \frac{1}{\rho^{2}} \mathcal{L}_{3} f^{\prime \prime}(t) e^{\rho t} d t \\
& \quad-\frac{1}{3 \rho} \mathcal{L}_{3} R(t) e^{\rho t}\left[\frac{3 f(t)}{\rho}+v(t, \rho)\right] d t .
\end{aligned}
$$

For fixed $\rho$ let $m=\max _{0 \leq x \leq 1}|v(x, \rho)|$; then

$$
\begin{aligned}
m & \leq \frac{1}{|\rho|^{2}}\left|\mathcal{L}_{3}\left[f^{\prime \prime}(t)+R(t) f(t)\right] e^{\rho t} d t\right|+\frac{1}{3|\rho|}\left|\mathcal{L}_{3} R(t) e^{\rho t} v(t, \rho) d t\right| \\
& \leq \frac{\mu_{1}}{|\rho|^{2}}+\frac{m \mu_{1}}{|\rho|} \leq \frac{\mu_{1}}{|\rho|^{2}}+\frac{m}{2},
\end{aligned}
$$

provided $|\rho| \geq 2 \mu_{2}$, where $\left|\mathcal{L}_{3} R(t) e^{\rho t} d t\right| \leq \mu_{2}$. Hence for such $\rho$ we have $m \leq 2 \mu_{1} /|\rho|^{2}$, and it follows that $v(x, \rho)=\rho^{-2} E_{1}(x, \rho)$.

It remains to show that $v^{\prime \prime}(x, \rho)=E_{2}(x, \rho)$. Differentiating (6), we have

$$
\begin{array}{r}
v^{\prime}(x, \rho)=-\frac{1}{\rho}\left\{\mathscr{L}_{2}\left[f^{\prime \prime}(t)+R(t) f(t)+\frac{1}{3 \rho} E_{1}(t, \rho)\right] e^{\rho t} d t\right\} \\
+\frac{E_{3}(x, \rho)}{\rho^{2}},
\end{array}
$$


where

$$
\begin{array}{r}
\mathcal{L}_{2} F(t) d t=e^{\omega_{1} \rho x} \int_{y}^{x} F(t) d t-\omega_{3} e^{\omega_{2} \rho x} \int_{y}^{-\omega_{2} x} F(t) d t \\
-\omega_{2} e^{\omega_{3} \rho x} \int_{y}^{-\omega_{3} x} F(t) d t,
\end{array}
$$

and we have used the fact that

$$
\begin{aligned}
\left|E_{1}\left(-\omega_{2} x, \rho\right)\right| & =\left|\rho^{2}\left(\Psi_{2}\left(-\omega_{2} x, \rho\right)-\frac{3 f\left(-\omega_{2} x\right)}{\rho}\right)\right| \\
& =\left|-\rho^{2} \omega_{3}\left(\Psi_{2}(x, \rho)-\frac{3 f(x)}{\rho}\right)\right|=\left|E_{1}(x, \rho)\right| .
\end{aligned}
$$

We can also show, as before in the case of the $\mathcal{L}_{3}$ operator, that if $|F(z)| \leq \mu$ on $|z| \leq 1$, then $\left|\mathcal{L}_{2} F(t) e^{\rho t} d t\right| \leq m_{2}$.

Differentiating (7), we obtain

$$
v^{\prime \prime}(x, \rho)=\mathcal{L}_{1}\left[f^{\prime \prime}(t)+R(t) f(t)+\frac{1}{3 \rho} E_{1}(t, \rho)\right] e^{\rho t} d t+\frac{E_{4}(x, \rho)}{\rho},
$$

where

$$
\begin{array}{r}
\mathcal{L}_{1} F(t) d t=e^{\omega_{1} \rho x} \int_{y}^{x} F(t) d t \\
+e^{\omega_{2} \rho x} \int_{y}^{-\omega_{2} x} F(t) d t \\
+e^{\omega_{3} \rho x} \int_{y}^{-\omega_{3} x} F(t) d t
\end{array}
$$

and we have used the fact that $\left|E_{1}^{\prime}\left(-\omega_{2} x, \rho\right)\right|=\left|E_{1}^{\prime}(x, \rho)\right|$ and that

$$
E_{1}^{\prime}(x, \rho)|=| \rho^{2} v^{\prime}(x, \rho)|\leq| \rho \mid M
$$

for $|\rho|$ sufficiently large.

Hence $v^{\prime \prime}(x, \rho)=E_{2}(x, \rho)$ since again $|F(z)| \leq \mu$ for $|z| \leq 1$ implies $\left|\mathcal{L}_{1} F(t) e^{\rho t} d t\right| \leq m_{1}$, and the proof of the lemma is complete.

3. Theorem. We proceed now to the proof of the following theorem.

THEOREM. If $f(x)=x^{2} \phi\left(x^{3}\right)$, where $\phi(z)$ is analytic on $|z| \leq 1$, the formal series for $f(x)$ converges uniformly to $f(x)$ on $0 \leq x \leq 1$. 
Proof. Since, for real $x$ and $\xi, u(x, \xi, \rho)$ is real for real $\rho$, by the principle of reflection we have $u\left(x, \xi, \rho^{*}\right)=[u(x, \xi, \rho)]^{*}$. This implies that the integrand in the expression for $I_{n}(x)$ given in Lemma 4 takes on values for $\rho$ on $\gamma_{n}^{\prime}=\gamma_{n} \cap S_{1}$ which are the complex conjugates of those it takes on for $\rho$ on $\gamma_{n}^{\prime \prime}=\gamma_{n} \cap S_{2}$. It suffices, then, to consider only the $\rho$ integration over $\gamma_{n}^{\prime}$. Denoting the result by $I_{n}^{\prime}(x)$, we have, by Lemmas 4 and 5 ,

$$
\begin{aligned}
I_{n}^{\prime}(x)=\frac{1}{2 \pi i} \int_{\gamma_{n}^{\prime}}\left\{\left[u(x, 0, \rho) \Psi_{1}(\rho)+\frac{3 f(x)}{\rho}+\frac{E_{1}(x, \rho)}{\rho^{2}}\right]\right. \\
\left.\quad \frac{u(x, 0, \rho)}{u^{\prime \prime}(1,0, \rho)}\left[u^{\prime \prime}(1,0, \rho) \Psi_{1}(\rho)+\frac{3 f^{\prime \prime}(x)}{\rho}+E_{2}(x, \rho)\right]\right\} d \rho
\end{aligned}
$$

and since, by Lemma 3, parts a) and c), we have

$$
\left|\frac{u(x, 0, \rho)}{u^{\prime \prime}(1,0, \rho)}\right| \leq \frac{M}{|\rho|^{2}}
$$

for $\rho$ on $\gamma_{n}^{\prime}$ and $n$ sufficiently large, we obtain

$$
I_{n}^{\prime}(x)=\frac{1}{2 \pi i} \int_{\gamma_{n}^{\prime}}\left[\frac{3 f(x)}{\rho}+\frac{E(x, \rho)}{\rho^{2}}\right] d \rho=\frac{f(x)}{2}+\epsilon_{n}^{\prime}(x),
$$

where

$$
\lim _{n \rightarrow \infty} \epsilon_{n}^{\prime}(x)=0
$$

uniformly in $x$. This proves the theorem.

At the expense of brevity, this theorem clearly could be generalized to problems involving somewhat more complicated boundary conditions and somewhat weaker analyticity conditions on $f(x), p(x)$, and $q(x)$; in connection with the latter contention, see [2].

\section{REFERENCES}

1. A. H. Flax, Aeroelastic problems at supersonic speed, Proc. Second International Aeronautics Conference, International Aeronautical Society, New York, 1949, pp. 322 - 360.

2. L. E. Ward, A third order irregular boundary value problem and the associated series, Trans. Amer. Math. Soc. 34 (1932), $417-434$.

3. G. Seifert, $A$ third order irregular boundary problem arising in aeroelastic wing theory, Quart. Appl. Math. 9 (1951), 210-218. 


\title{
PACIFIC JOURNAL OF MATHEMATICS
}

\section{EDITORS}

\section{R. M. RobINSON \\ University of California \\ Berkeley 4, California}

\author{
*R. P. Dilwor TH \\ California Institute of Technology \\ Pasadena 4, California
}

\author{
E. F. BECKENBACH, Managing Editor \\ University of California \\ Los Angeles 24, California
}

${ }^{*}$ During the absence of Herbert Busemann in 1952.

\section{ASSOCIATE EDITORS}

$\begin{array}{llll}\text { R. P. DILWORTH } & \text { P. R. HALMOS } & \text { B } \emptyset \text { RGE JESSEN } & \text { J. J. STOKER } \\ \text { HERBERT FEDERER } & \text { HEINZ HOPF } & \text { PAUL LÉVY } & \text { E. G. STRAUS } \\ \text { MARSHALL HALL } & \text { R. D. JAMES } & \text { GEORGE PÓLYA } & \text { KÖSAKU YOSIDA }\end{array}$

\section{SPONSORS}

UNIVERSITY OF BRITISH COLUMBIA

CALIFORNIA INSTITUTE OF TECHNOLOGY

U NIVERSITY OF CALIFORNIA, BERKELEY

UNIVERSITY OF CALIFORNIA, DAVIS

UNIVERSITY OF CALIFORNIA, LOS ANGELES

UNIVERSITY OF CALIFORNIA, SANTA BARBARA

OREGON STATE COLLEGE

UNIVERSITY OF OREGON

\author{
UNIVERSITY OF SOUTHERN CALIFORNIA \\ STANFORD UNIVERSITY \\ WASHINGTON STATE COLLEGE \\ UNIVERSITY OF WASHINGTON \\ AMERICAN MATHEMATICAL SOCIETY \\ NATIONAL BUREAU OF STANDARDS, \\ INSTITUTE FOR NUMERICAL ANALYSIS
}

Mathematical papers intended for publication in the Pacific Journal of Miathematics should be typewritten (double spaced), and the author should keep a complete copy. Manuscripts may be sent to any of the editors. All other communications to the editors should be addressed to the managing editor, E. F. Beckenbach, at the address given above.

Authors are entitled to receive 100 free reprints of their published papers and may obtain additional copies at cost.

The Pacific Journal of Mathematics is published quarterly, in March, June, September, and December, by the University of California, Berkeley 4, California. The price per volume (4 numbers) is $\$ 8.00$; single issues, $\$ 2.50$. Special price to individual faculty members of supporting institutions and to individual members of the American Mathematical Society: $\$ 4.00$ per volume; single issues, $\$ 1.25$.

Subscriptions, orders for back numbers, and changes of address should be sent to the publishers, University of California Press, Berkeley 4, California.

Printed at Ann Arbor, Michigan. Entered as second class matter at the Post Office, Berkeley, California.

\section{UNIVERSITY OF CALIFORNIA PRESS • BERKELEY AND LOS ANGELES}




\section{Pacific Journal of Mathematics}

\section{Vol. 2, No. $3 \quad$ March, 1952}

Lars V. Ahlfors, Remarks on the Neumann-Poincaré integral equation .... 271

Leonard P. Burton, Oscillation theorems for the solutions of linear, nonhomogeneous, second-order differential systems ............ 281

Paul Civin, Multiplicative closure and the Walsh functions . . . . . . . . . . . 291

James Michael Gardner Fell and Alfred Tarski, On algebras whose factor algebras are Boolean .................................. 297

Paul Joseph Kelly and Lowell J. Paige, Symmetric perpendicularity in Hilbert geometries .................................. 319

G. Kurepa, On a characteristic property of finite sets .............. 323

Joseph Lehner, A diophantine property of the Fuchsian groups ......... 327

Donald Alan Norton, Groups of orthogonal row-latin squares ........... 335

R. S. Phillips, On the generation of semigroups of linear operators ....... 343

G. Piranian, Uniformly accessible Jordan curves through large sets of relative harmonic measure zero ........................ 371

C. T. Rajagopal, Note on some Tauberian theorems of $O . S z \tilde{A} ; s z \ldots \ldots \ldots 377$

Halsey Lawrence Royden, Jr., A modification of the Neumann-Poincaré method for multiply connected regions .................... 385

George H. Seifert, A third order irregular boundary value problem and the associated series ...................................... 395

Herbert E. Vaughan, Well-ordered subsets and maximal members of ordered sets....

Hans F. Weinberger, An optimum problem in the Weinstein method for eigenvalues.

Shigeki Yano, Note on Fourier analysis. XXXI. Cesàro summability of Fourier series. 